\title{
History and Flight Development of the Electrodynamic Dust Shield
}

Michael R. Johansen, Paul J. Mackey, Michael D. Hogue, Rachel E. Cox, James R. Phillips III, and Carlos I. Calle

National Aeronautics and Space Administration, Kennedy Space Center 


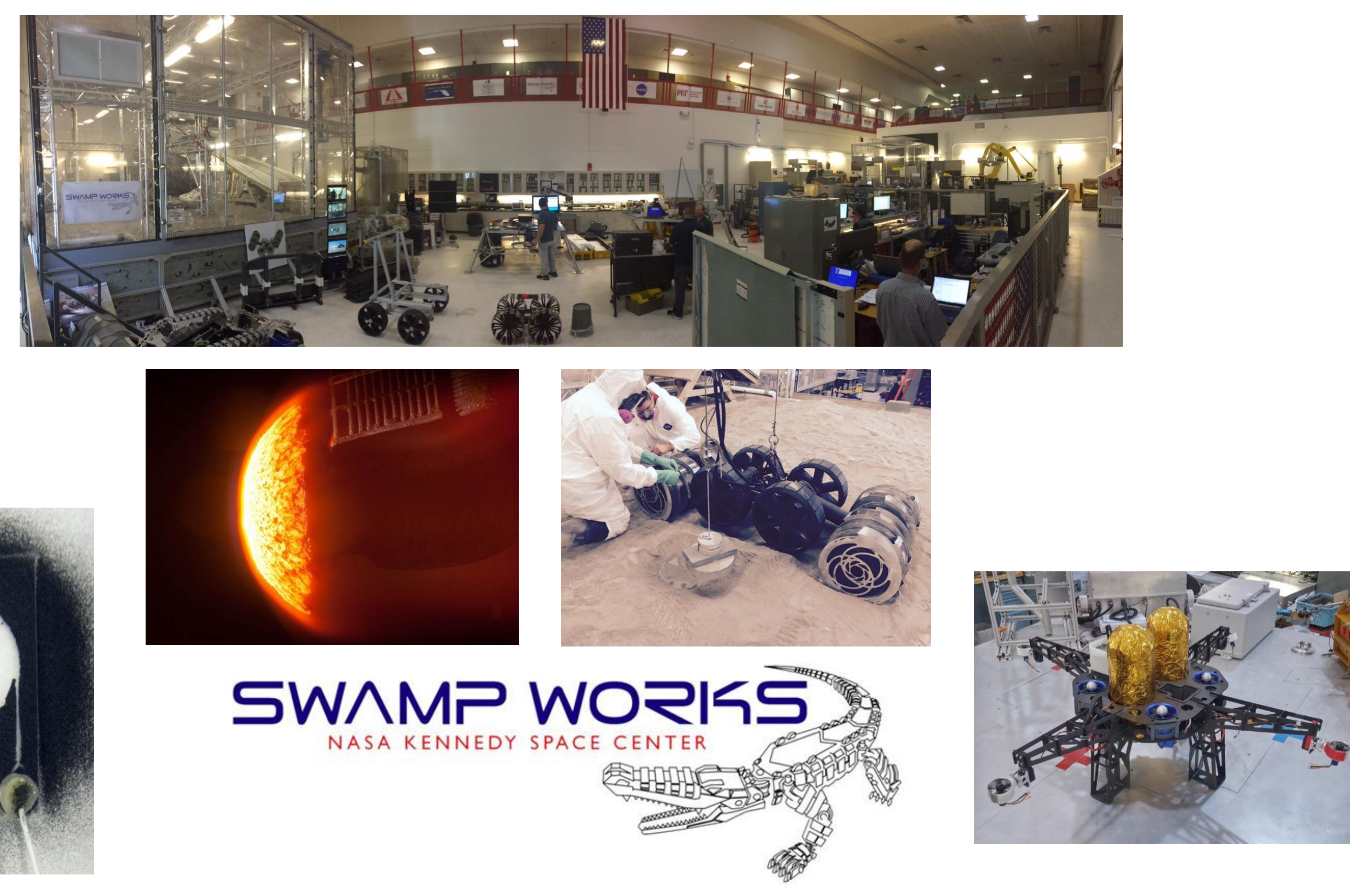




\section{The Dust Problem}

- Dust from this equipment fell back onto the lower crewmember and into the cabin and seemed to bind the conveyor so as to require considerable force to operate it. - Apollo 11 Mission Report

- The cohesive properties of lunar dust in a vacuum, augmented by electrostatic properties, tend to make it adhere to anything it contacts.Apollo 12 Mission Report

- After exposure to a dusty lunar environment, the both crewmen's suit wrist-ring disconnects were hard to rotate to the locked and unlocked position. - Apollo 16 Mission Report

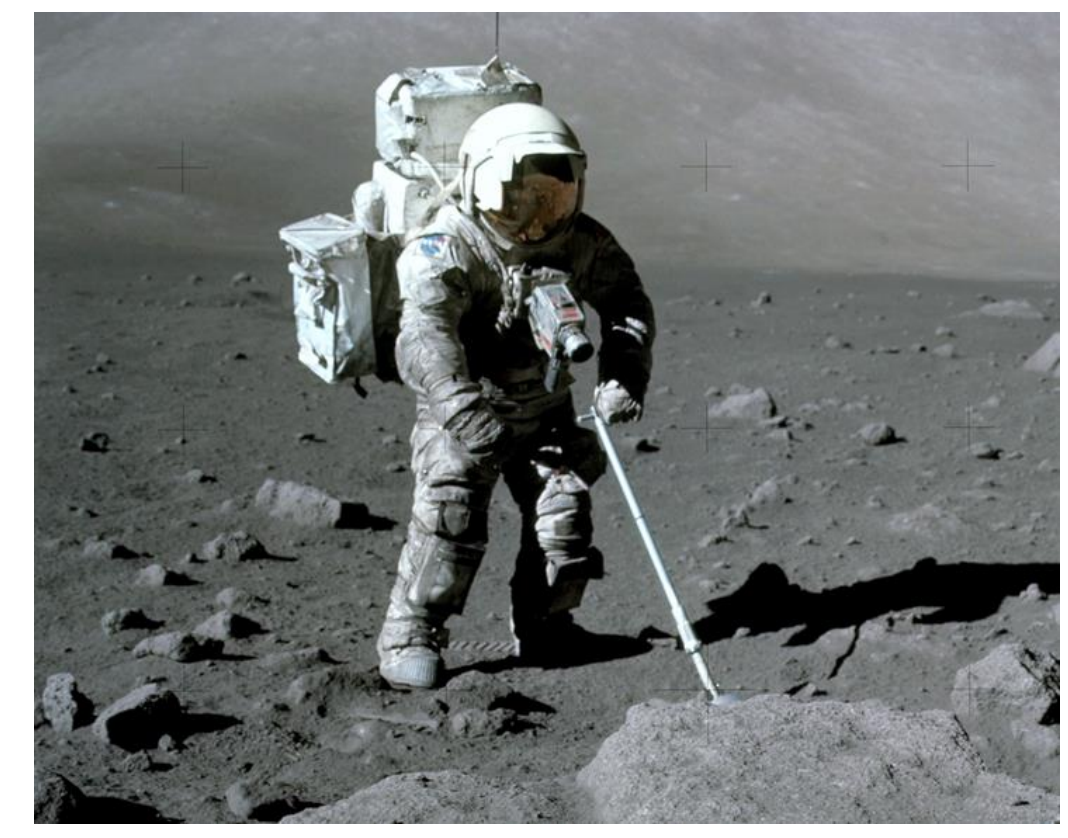

SW 
- Apollo 16 Moon Buggy Video 


\section{Electrodynamic Dust Shield}

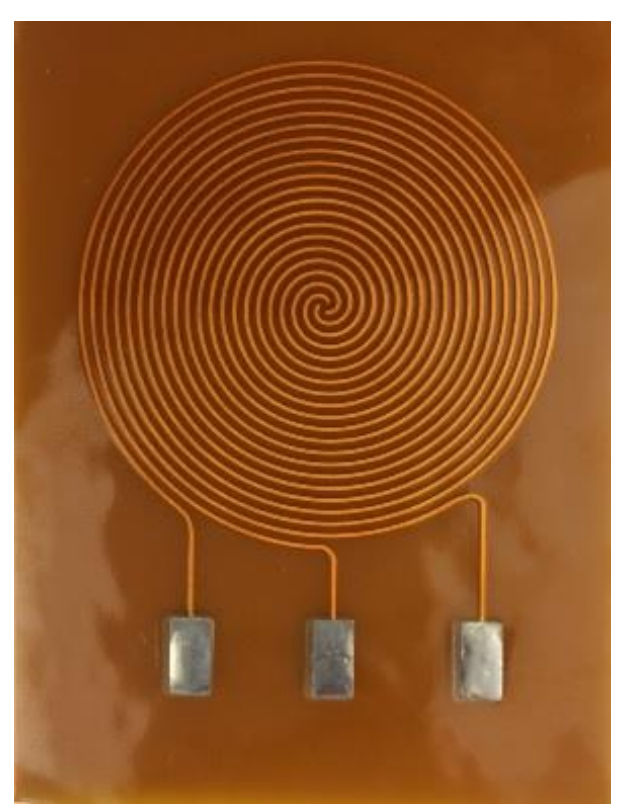

Cu/Kapton

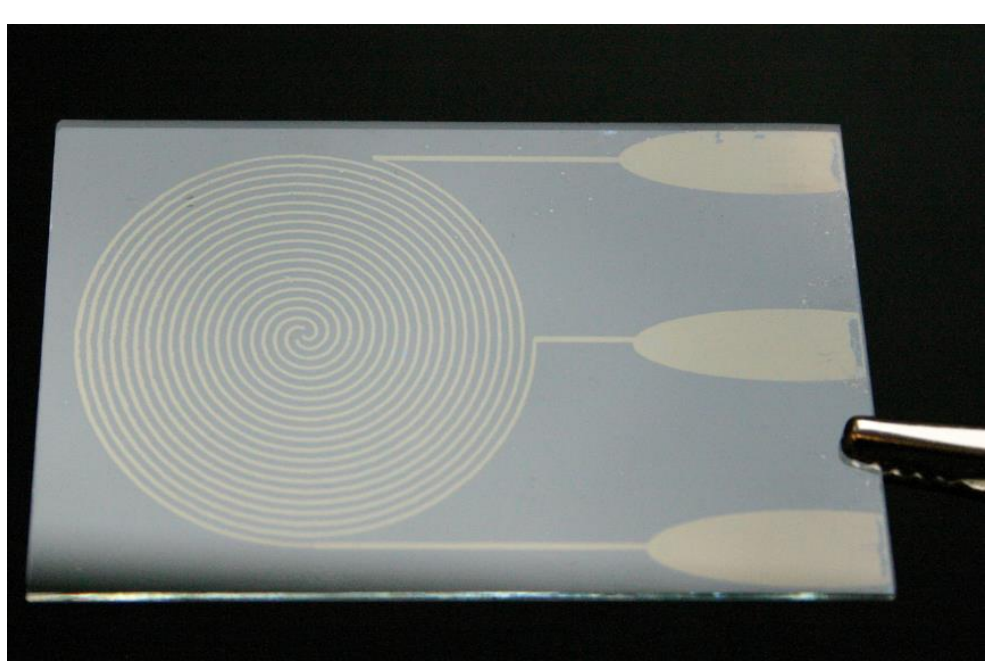

ITO/glass

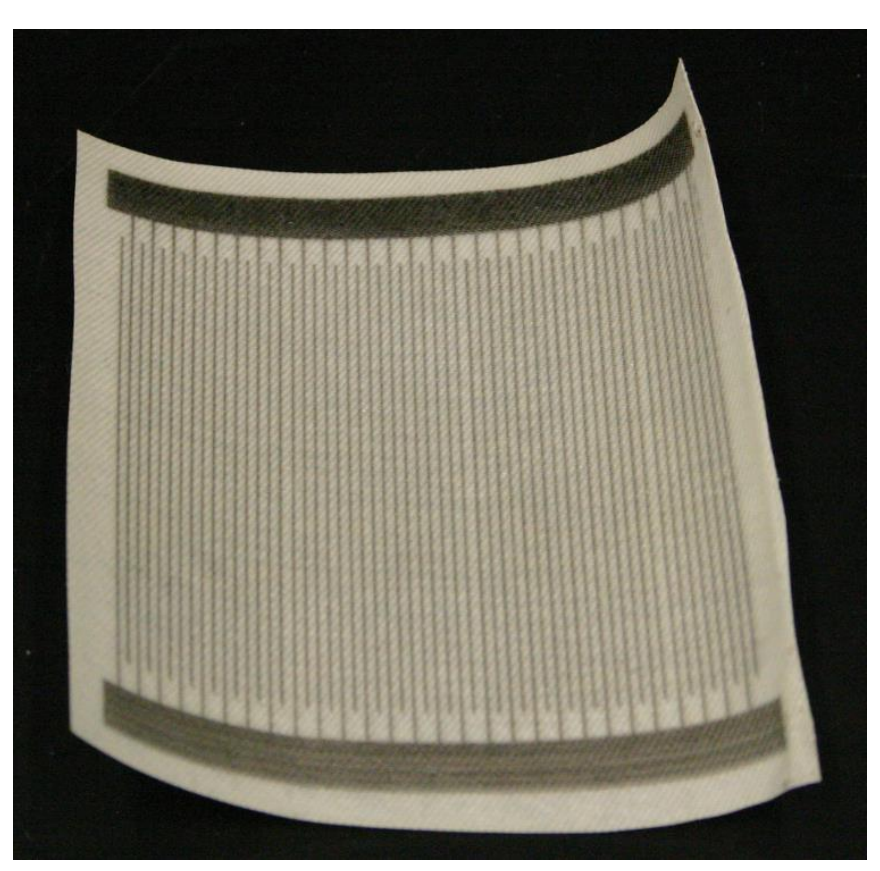

CNT/fabric

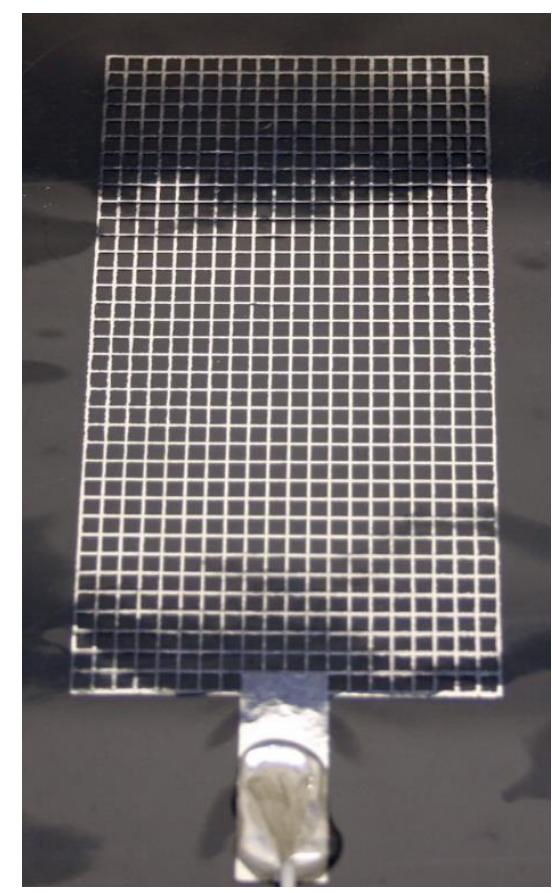

$\mathrm{Ag} / \mathrm{FEP}$

SWAMP WORKS 
- EDS Operation Video 


\section{Testing}

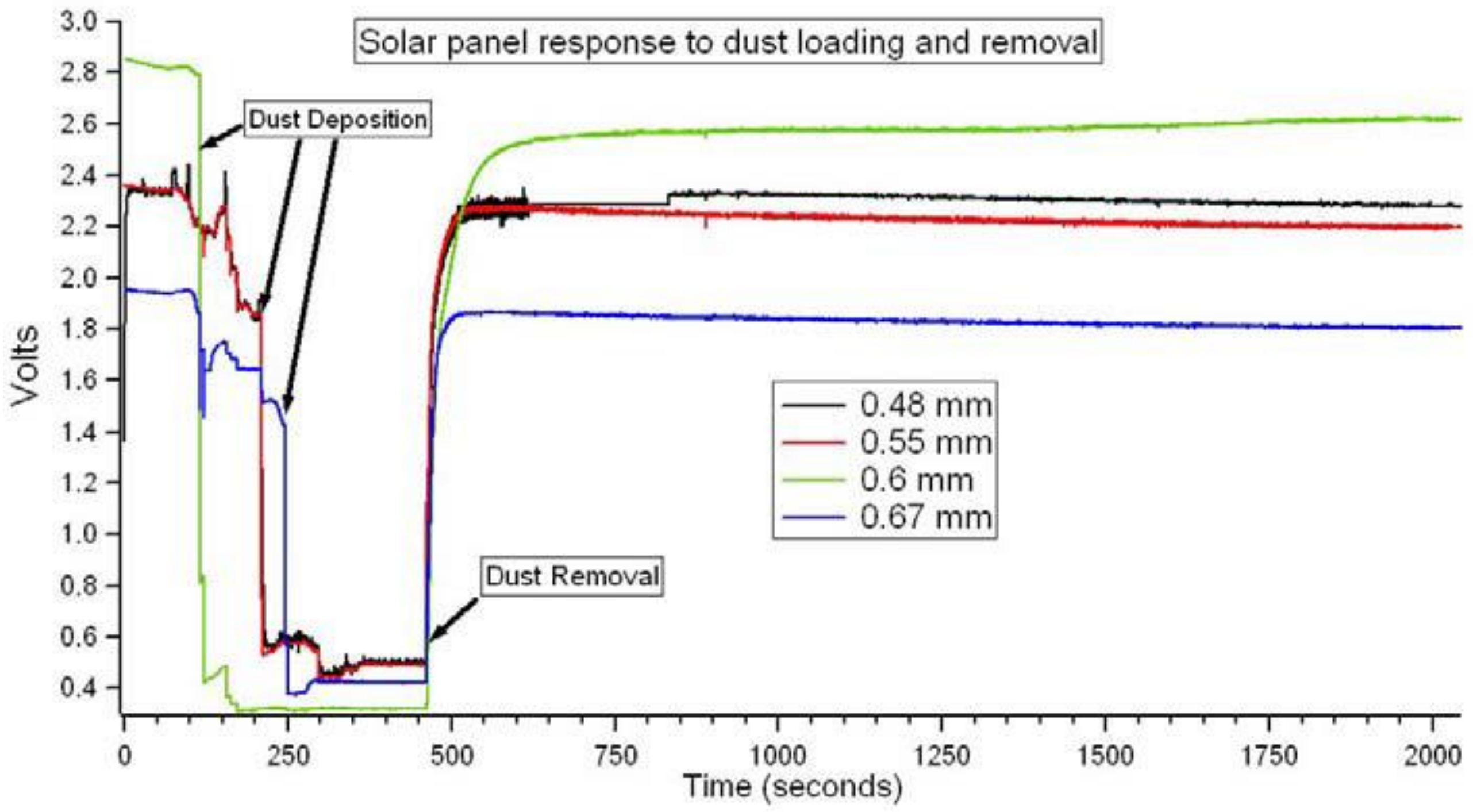




\section{Testing}

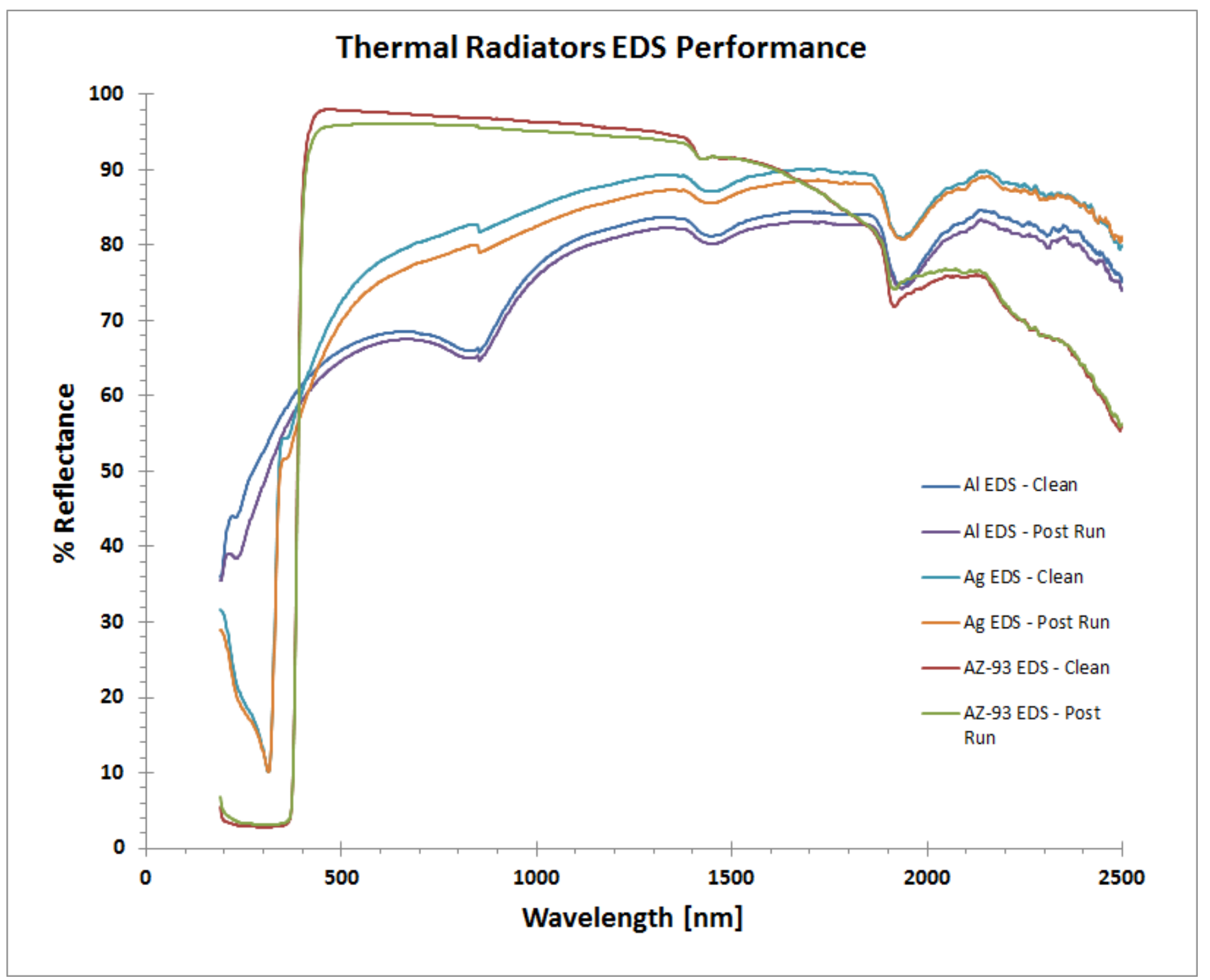


- RGF Video 


\section{MISSE}

- Materials International Space Station Experiment

- Designed to test material response

- Atomic Oxygen

- Radiation

- Vacuum

- Thermal cycling

- Data from power supply and photos used to determine shield health

- Currently work - EDS with space compatible materials
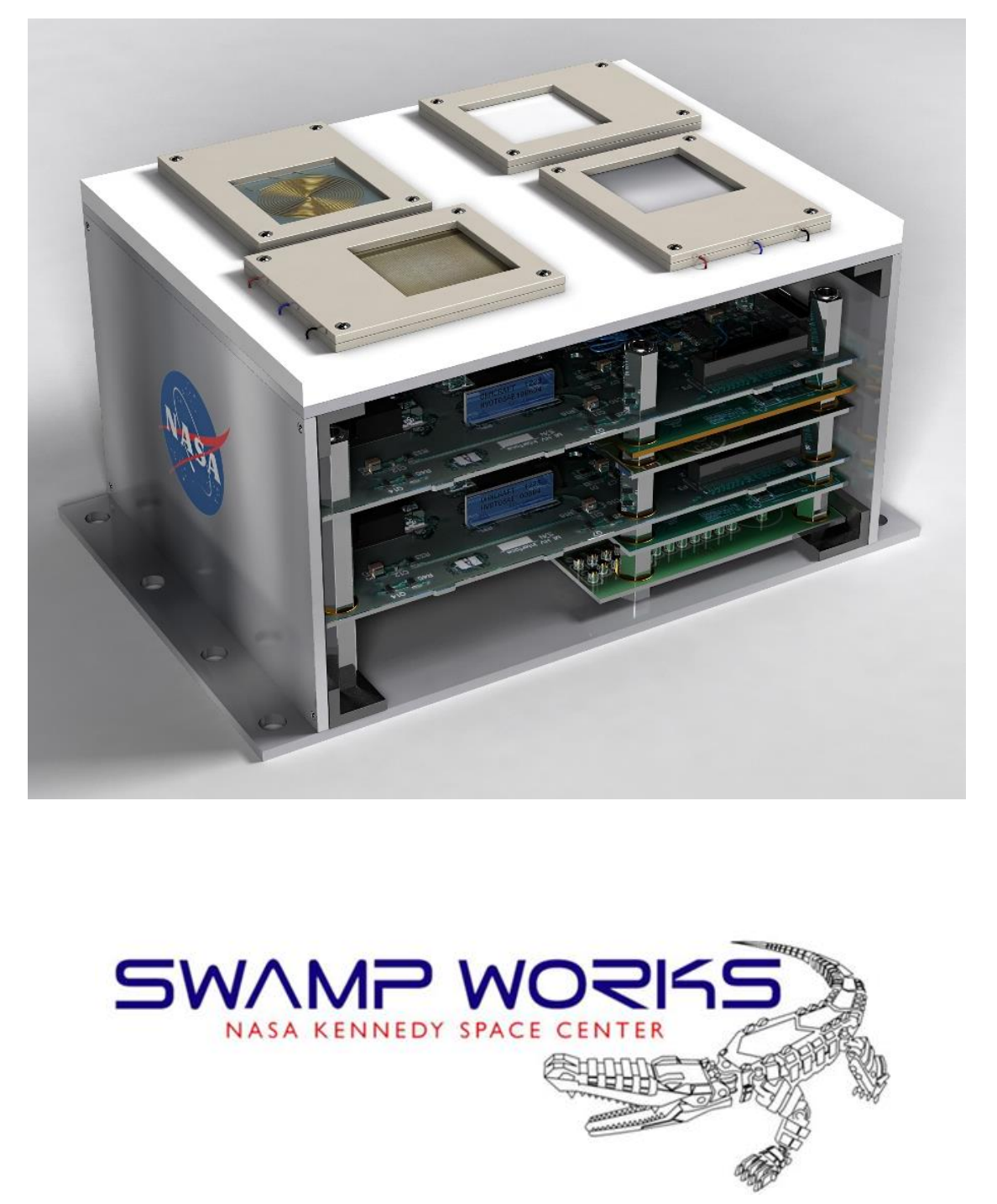


\section{Lunar CATALYST}

- Lunar Cargo Transportation and Landing by Soft Touchdown

- NASA provides

- Technical expertise

- Test facilities

- Loan equipment/software

- Mission concept - Fly EDS and electronics on footpad

- Current work - Reduce electronics footprint

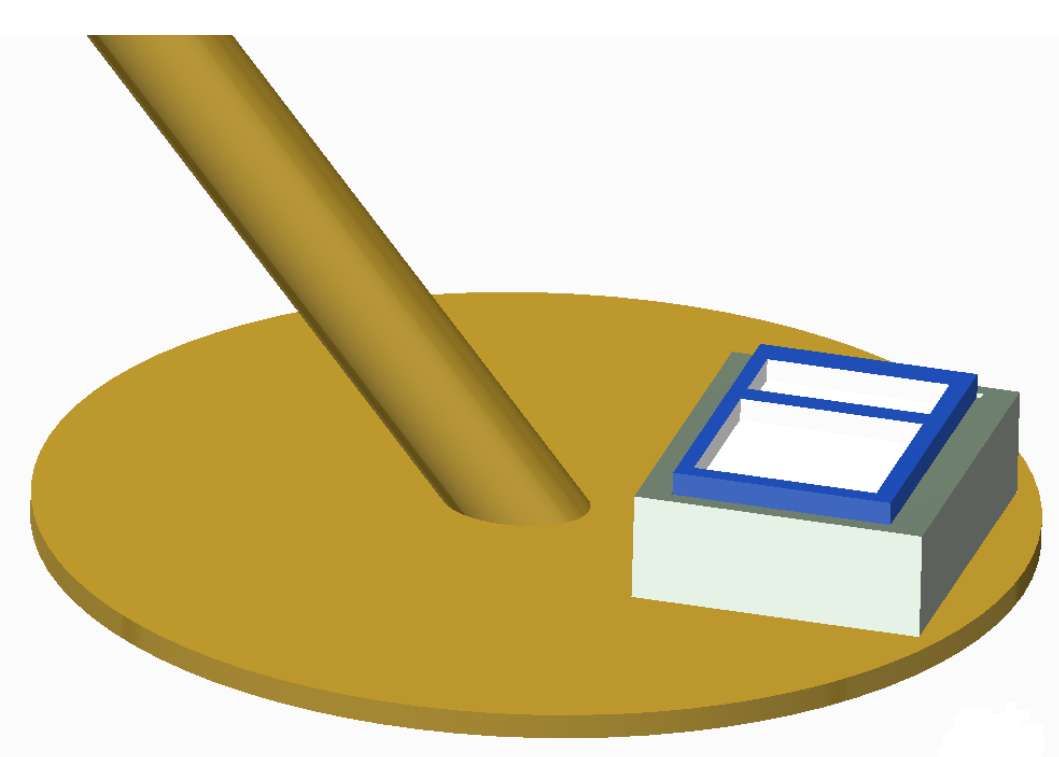



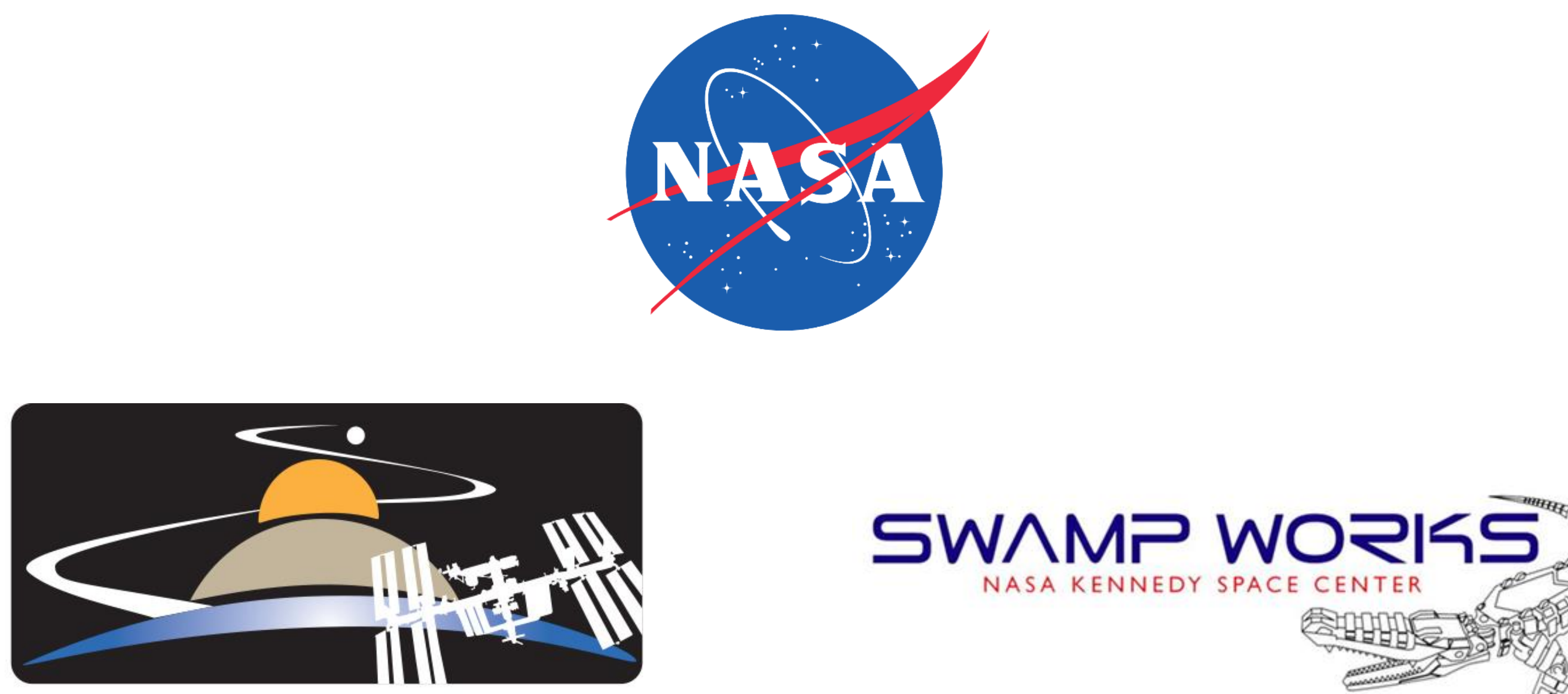

NASA KENNEDY SPACE CENTER

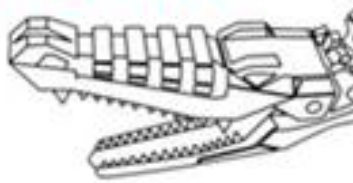

Exploration Research and Technology Programs 